Research report

\title{
Automatic detection and sonification of nonmotor generalized onset epileptic seizures: Preliminary results
}

\author{
Lorenzo Frassineti $^{\mathrm{a}, *}$, Carmen Barba ${ }^{\mathrm{b}}$, Federico Melani ${ }^{\mathrm{b}}$, Francesca Piras ${ }^{\mathrm{b}}$, Renzo Guerrini ${ }^{\mathrm{b}, \mathrm{c}}$, \\ Claudia Manfredi ${ }^{\mathrm{a}}$ \\ ${ }^{a}$ Department of Information Engineering, Università degli Studi di Firenze, Firenze, Italy \\ ${ }^{\mathrm{b}}$ Paediatric Neurology Unit, Neuroscience Department, Children's Hospital A. Meyer - University of Florence, Firenze, Italy \\ ${ }^{\mathrm{c}}$ IRCCS Stella Maris Foundation, Pisa, Italy
}

\section{H I G H L I G H T S}

- A diagnostic support is presented to speed up and facilitate the interpretation of EEG signals.

- Pre-processing is based on the Stationary Wavelet Transform for artefacts reduction.

- Sonification techniques are developed significantly reducing false positive alarms.

- We investigated a specific seizure type: absence seizures.

- Results show high accuracy (about 96\%) and low latency time (1.25 s on average).

\section{A R T I C L E I N F O}

\section{Keywords:}

EEG

Early seizure detection

Denoising

SWT

Absence seizure

Sonification

\begin{abstract}
A B S T R A C T
Long-term video-EEG monitoring has improved diagnosis and treatment of epilepsy, especially in children. However, the amount of data neurophysiologists must analyze has grown remarkably.

The main purpose of this paper is to provide a diagnostic support to speed up and ease EEG interpretation for a specific application concerning absence seizures, a type of non-motor generalized epileptic seizures.

The proposed method consists of a pre-processing step where signals are filtered through the Stationary Wavelet Transform for the reduction of possible artefacts. Subsequently, a supervised automatic classification method is implemented for seizure detection, based on the Support Vector Machine Fine Gaussian method. Finally, a post-processing step is implemented in which spatial and temporal thresholds are defined for both online and offline application.

In addition, a method that applies sonification techniques is developed. Sonification techniques could speed up the process of interpreting information, allowing rapid clinical intervention and a continuous monitoring of the event.

The dataset consists of 30 EEG recordings performed in 24 children with absence seizures, clinically evaluated at the Meyer Children's Hospital in Firenze, Italy.

The method shows encouraging results both in terms of balanced accuracy (about 96\%) and latency times (1.25 s on average), which might make it suitable for online clinical trials. In fact, it was implemented in the perspective of a possible real-time application in clinical practice.
\end{abstract}

\section{Introduction}

Electroencephalography (EEG), it the most widely used technique for the diagnosis and evaluation of epileptic seizures. EEG recordings, lasting from 15 to $30 \mathrm{~min}$ (short monitoring) to more than $24 \mathrm{~h}$ (longterm monitoring), allow defining background activity while awake, sleep EEG patterns, as well as identify epileptiform abnormalities and possibly capture seizures. Routine scalp EEG recordings are commonly performed using 19-21 electrodes placed according to the 10-20 system. Therefore, the clinician is required to simultaneously analyze a considerable amount of data.

In clinical practice, offline automatic detection methods have

\footnotetext{
* Corresponding author.

E-mail address: lorenzo.frassineti42@gmail.com (L. Frassineti).
} 
already been proposed and implemented, such as systems assessing quantitative EEG (qEEG) (Swisher, 2017).

The clinical usefulness of an online tool for automatic detection of epileptic seizures and epileptiform abnormalities is still a matter of debate (Vidyaratne and Iftekharuddin, 2017). If implemented, such a tool could be valuable in clinical neurological practice, especially when a real-time seizure detection is required to provide an early and effective treatment (Temko et al., 2017). On the other hand, the abovementioned tool might also be useful in the off-line analysis of video-EEG recordings, which is a high-cost- and time-consuming procedure, in order to help interpreters in the rapid and correct diagnosis and management of epilepsy and paroxysmal events.

In the last years, there has been a growing scientific interest in methodological results concerning early seizure detection. New methodologies must consider both the accuracy of the identification system and the latency time required to process a decision by the system, which is of basic relevance for the online or real-time application of the method. Indeed, current limitations are represented by a poor balance between high accuracy and low latency times.

As reported in a recent review on EEG scalp analysis Alotaiby et al. (2014); Alam (2013) obtained 100\% performance in terms of accuracy, but the estimated latency time was of $23.6 \mathrm{~s}$. To solve the same problem in a complementary way, Yoo (2013) used EEG time windows of only $2 \mathrm{~s}$ of duration, but reached only $84.4 \%$ of accuracy. More recently, Vidyaratne and Iftekharuddin (2017) obtained an accuracy of $99.8 \%$ for short-monitoring and an average latency time of about $1.89 \mathrm{~s}$.

In this paper, we have been concerned with achieving the best performance in terms of both accuracy and latency for online application but did not compare our method to those mentioned above, as they investigate different types of seizures. Indeed, high latency time would not allow a clinical evaluation of the seizures in real time and low accuracy would produce an overload of information to be analyzed. We did not consider seizure detection as a whole, but focused on absence seizures, a specific type of non-motor generalized seizures Fisher et al. (2017) with abrupt onset and offset of altered awareness with limited or no motor manifestations. Absences can vary in frequency and duration in relation to specific syndromes and consist of a sudden break of awareness with blank staring and speech arrest or unresponsiveness. Fine movements of the eyelids or upward eye deviation can at times be observed. As a rule, the patient does not fall to the ground or experience changes in posture and can also continue simple motor activities. After two to a few tens of seconds the subject re-establishes full contact with the environment and resumes previous activities. Memory of events occurring during the seizures is usually impaired, although there may be some retained awareness, particularly in adolescents. These seizures can appear a few times up to hundreds of times a day, to the point that the person's attention is severely disrupted.

Absences are a typical childhood disorder, concerning $10-17 \%$ of all epilepsy patients in this age range (Zeng, 2016) in the context of genetic generalized epilepsies. In typical absences, EEG shows generalized spike-wave discharges at a frequency higher than $2.5 \mathrm{~Hz}$. Conversely, atypical absences are characterized by a more gradual onset or termination or significant changes in tone supported by atypical, usually slow, generalized spike and wave discharges, at less than $2.5 \mathrm{~Hz}$, in the EEG Fisher et al. (2017).

Scalp EEG signals are usually strongly masked by several artefacts and one of the major challenges is finding a method to cut down the contribution of spurious elements.

In recent years, several methods have been proposed to eliminate or reduce artefacts of various kinds. Most methods are based on Blind Source Separation (BSS) algorithms (Md Kafiul Islam et al., 2016), including the Independent Component Analysis (ICA) (Zhu, 2008; Hamaneh, 2014). These approaches have shown good results in processing large amounts of data. The main limitations are represented by the hypotheses made a priori: first, the linear independence between noise and signal, which is not always verified; secondly, the presence of artefacts that may be observed only in a limited number of channels, as well as the presence of the epileptic event.

Another methodology for processing artefacts is represented by the adaptive filters widely used in biomedical signals (Abd Rahman, 2015). The main problem of such methods is that they require an artefact reference signal (like EMG, EOG, ECG etc), which is not always available, and therefore they are usually limited to the removal of a single type of artefact (Boudet et al., 2012; Molla, 2012). There are also adaptive filters that do not need an artefact reference signal, such as the Wiener filter (Urigüen, 2015), but they require a calibration step to define the necessary number of iterations for a correct artefact removal, that cannot be performed in real time.

Another category is represented by methods belonging to the Source Decomposition class, i.e. systems that extract information by breaking down the signal into a series of elementary waveforms. Methods using tools such as Wavelets are a well-known example of this methodology (Patel, 2014; Md Kafiul Islam et al., 2016; Chavez, 2018). Common limitations are the choice of the mother wavelet and the number of decomposition levels. Indeed, as the method is based on the evaluation of a degree of similarity between the signal and the wavelet function, the selection of the mother wavelet is a crucial point for the correct use of the methodology. Therefore, the choice of the "best" wavelet function among all those proposed over the years (Hramov, 2015) is still a challenge.

There are several reasons why it may be useful to turn graphical and/or numerical information into sounds: overload of visual information; providing a support to visual information; speeding up the interpretation of information processes for real-time or online applications (Loui, 2014; Temko et al., 2015). The commonly accepted definition of sonification comes from G. Kramer et al. (Kramer et al., 1999): "Sonification is the transformation of data relations into perceived relations in an acoustic signal for the purposes of facilitating communication or interpretation".

For a correct sonification procedure, it is essential to develop a method that allows discriminating between the relevant information and artefacts in almost real time.

Another possible obstacle to the use of these techniques is inherent in the sound information: unless properly coded, the interpretation of perceived sounds is highly subjective. Thus, a further criterion applied in this work is the production of easily recognizable and reproducible sounds.

In this paper we propose a system optimized for automatic recognition and online sonification of absence seizures during scalp EEG recordings, which might allow a fast detection and interpretation of useful information, significantly reducing false positive alarms.

\section{Results}

The performance of the proposed method, that will be described in Section 4, was tested on 30 EEG recordings from a set of 24 children (8 males, 16 females, age range $4-18$ years, mean $8.7 \pm 3.8$ years) with absence seizures evaluated at the Paediatric Neurology Unit, Children's Hospital A. Meyer, Firenze, Italy. Full clinical and EEG information concerning these patients is summarized in Table 1 .

Specifically, only one recording per patient was considered in 21 out of 24 patients, while three recordings were analysed for patients 8,13 and 22. In Table 1 only the date of the first recording is reported.

Written informed consent for using video-EEG data for research aims was obtained from all patients (or guardians of patients). All data were retrospectively analyzed, with no impact on diagnostic and treatment strategies.

Absence seizures were typical in 20 children, atypical in two of them and with a myoclonic component in the remaining two. Representative ictal EEG traces were retrospectively selected within the whole Meyer Hospital Pediatric Neurology database by expert neurophysiologists, based on the recording of nonmotor generalized onset 
Table 1

Clinical and EEG information on the 24 patients included in the study.

\begin{tabular}{|c|c|c|c|c|c|c|c|c|c|}
\hline \multicolumn{10}{|c|}{ Clinical and EEG Information on the 24 patients include in the study } \\
\hline Patient code & $\begin{array}{l}\text { Epilepsy } \\
\text { classification }\end{array}$ & $\begin{array}{l}\text { Absence } \\
\text { classification }\end{array}$ & $\begin{array}{l}\text { Age at seizure } \\
\text { onset/age at last } \\
\text { seizure (ys) }\end{array}$ & $\begin{array}{l}\text { Age at } \\
\text { EEG } \\
\text { (ys) }\end{array}$ & Ictal EEG & AED tried & $\begin{array}{l}\text { Seizure free at } \\
\text { last FU (yes/ } \\
\text { no) }\end{array}$ & $\begin{array}{l}\text { AED withdrawal at } \\
\text { last FU (yes/no) }\end{array}$ & $\begin{array}{l}\text { Number of } \\
\text { analyzed } \\
\text { recordings }\end{array}$ \\
\hline 1 & $\mathrm{CAE}$ & Typical & $4.9 / 6$ & 5 & 3 HZ SW & ESM, VPA & Yes & No & 1 \\
\hline 2 & GGE & Typical & $5 /$ still present & 13 & $3 \mathrm{HZ} \mathrm{SW}$ & $\begin{array}{l}\text { LTG, PB, VPA, } \\
\text { ESM, ZNS, LEV, } \\
\text { TPM, steroids }\end{array}$ & No & No & 1 \\
\hline 3 & GGE & Atypical & $11 /$ still present & 13 & $\begin{array}{l}\text { Polyspikes } \\
\text { followed by SW }\end{array}$ & $\begin{array}{l}\text { ESM, VPA, PB, } \\
\text { CLZ, CNZ, STP, } \\
\text { RFN, LEV, } \\
\text { steroids }\end{array}$ & No & No & 1 \\
\hline 4 & GGE & Typical & $5 /$ still present & 18 & $3 \mathrm{HZ} \mathrm{SW}$ & ESM & No & No & 1 \\
\hline 5 & GGE & Typical & $4.8 / 7.5$ & 6 & 3 HZ SW & VPA, ESM & Yes & No & 1 \\
\hline 6 & $\mathrm{CAE}$ & Typical & $6 / 7$ & 6 & 3 HZ SW & VPA, ESM & Yes & Yes & 1 \\
\hline 7 & $\mathrm{CAE}$ & Typical & $2 / 5$ & 4 & $3 \mathrm{HZ} \mathrm{SW}$ & VPA, ESM, LTG & Yes & Yes & 1 \\
\hline 8 & GGE & Typical & $9.8 / 10.2$ & 10 & $3 \mathrm{HZ} \mathrm{SW}$ & ESM & Yes & No & 3 \\
\hline 9 & CAE & Typical & $5 / 6$ & 6 & 3 HZ SW & VPA & Yes & Yes & 1 \\
\hline 10 & GGE & Atypical & $6 /$ still present & 9 & $2.5 \mathrm{HZ} \mathrm{SW}$ & VPA, ESM & No & No & 1 \\
\hline 11 & $\mathrm{CAE}$ & Typical & $5.6 / 7.2$ & 7 & 3 HZ SW & ESM, VPA & Yes & Yes & 1 \\
\hline 12 & CAE & Typical & $5.5 / 6$ & 6 & $3 \mathrm{HZ} \mathrm{SW}$ & ESM & Yes & No & 1 \\
\hline 13 & GGE & Typical & $6.6 / 7.4$ & 7 & $3 \mathrm{HZ} \mathrm{SW}$ & VPA & Yes & No & 3 \\
\hline 14 & CAE & Typical & $10 / 12$ & 11 & 3 HZ SW & ESM & Yes & No & 1 \\
\hline 15 & CAE & Typical & $6.5 / 7.1$ & 6.9 & 3 HZ SW & VPA & Yes & No & 1 \\
\hline 16 & CAE & Typical & $7.6 / 8.1$ & 8 & 3 HZ SW & VPA & Yes & Yes & 1 \\
\hline 17 & CAE & Typical & $9 / 10$ & 9 & 3 HZ SW & ESM & Yes & No & 1 \\
\hline 18 & JAE & Typical & $13 / 17$ & 17 & $3 \mathrm{HZ} \mathrm{SW}$ & VPA & Yes & Yes & 1 \\
\hline 19 & $\mathrm{CAE}$ & Typical & $7.3 / 8$ & 7.8 & 3 HZ SW & VPA, ESM & Yes & Yes & 1 \\
\hline 20 & CAE & Typical & $5 / 6$ & 5 & $3 \mathrm{HZ} \mathrm{SW}$ & VPA, ESM & Yes & Yes & 1 \\
\hline 21 & CAE & Typical & $6.5 /$ still present & 7 & 3 HZ SW & VPA, ESM & No & No & 1 \\
\hline 22 & GGE & Typical & $5.5 / 7$ & 7 & $3 \mathrm{HZ} \mathrm{SW}$ & VPA, ESM & Yes & No & 3 \\
\hline 23 & $\mathrm{CAE}$ & Typical & $4 / 4.2$ & 4 & $3 \mathrm{HZ} \mathrm{SW}$ & ESM & Yes & No & 1 \\
\hline 24 & CAE & Typical & $4.5 / 5.1$ & 5 & 3 HZ SW & ESM, VPA & Yes & No & 1 \\
\hline
\end{tabular}

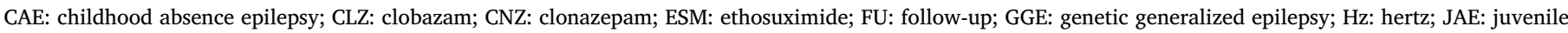

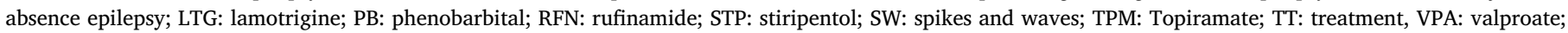
YS: years; ZNS: zonisamide.

seizures (either typical or atypical), without taking in account additional clinical information.

The length of the signals was $53 \pm 18$ s for each channel. EEG was recorded using 19 channels placed according to the international standard 10-20 system (longitudinal bipolar montage). All the derivations are considered in this study. Signals were sampled at $256 \mathrm{~Hz}$ and filtered in the frequency band $1.6 \mathrm{~Hz}-32 \mathrm{~Hz}$ for visual analysis.

In addition, the method was tested considering the whole duration of the signals, that is $47 \pm 10 \mathrm{~min}$ for each EEG channel.

The occurrence of "absence seizures" was marked by expert neurophysiologists. In all channels the beginning and the end of absence seizures were annotated, and the other epochs were considered as "interictal".

For the training and validation phase, 6 randomly selected recordings were used corresponding to patients 4, 6, 7, 12 and the first recording of patients 13 and 22, while the remaining 24 recordings were considered for the test phase. This subdivision allowed evaluating possible inter-physiological variations.

On the validation set a $\mathrm{F} 1_{\text {score }}$ equal to $93 \%$ was obtained. This result is in line with recent literature, where the Support Vector Machine (SVM) classifiers showed the best performances for binary classification of epileptic seizures (Upadhyay, 2016).

Table 2 shows the results obtained on the 24 test signals. $\mathrm{F} 1_{\text {score, }}$, Balanced Accuracy (BACC) and Matthews Correlation Coefficient (MCC) are compared. Methodological details are reported in Section 4.2.4. Results are obtained with Fine Gaussian Support Vector Machine (FGSVM), filtering with Stationary Wavelet Transform (SWT with Bior 3.3) using the Haar wavelet Md Kafiul Islam et al. (2016) and without filtering (No Filter).

Table 3 shows the results on the same test-set with the Bior 3.3
Table 2

Performance of FGSVM filtering with Bior 3.3, Haar wavelet and without filtering. Results are obtained using a test set of 24 recordings.

\begin{tabular}{lllll}
\hline & $\begin{array}{l}\text { Time duration } \\
{[\mathrm{s}]}\end{array}$ & BACC & $\mathrm{F} 1_{\text {score }}$ & \multicolumn{1}{l}{ MCC } \\
\hline Bior 3.3 & $53 \pm 18$ & $93.0 \% \pm 4.6 \%$ & $89.4 \% \pm 5.8 \%$ & $0.86 \pm 0.07$ \\
Haar & $53 \pm 18$ & $88.0 \% \pm 4.6 \%$ & $82.3 \% \pm 8.7 \%$ & $0.78 \pm 0.08$ \\
No-filter & $53 \pm 18$ & $64.9 \% \pm 10.8 \%$ & $43.0 \% \pm 16.5 \%$ & $0.40 \pm 0.11$ \\
\hline
\end{tabular}

filtering method, comparing the following classifiers: FGSVM, k-Nearest Neighbours (KNN, $\mathrm{k}=1$ ), Boosted Tree and Logistic Regression. They were chosen both because they gave high values of $\mathrm{F} 1_{\text {score }}$ in the validation set and because they are among the most used ones in the literature (Alotaiby et al., 2014; Upadhyay, 2016).

Finally, Table 4 shows the comparisons between the average performance with FGSVM obtained in online (with spatial threshold, ONLINE) and offline mode (with spatial and temporal thresholds, OFFLINE) with respect to that with automatic recognition only (FGSVM Bior 3.3). The test set is made of the same group of 24 recordings as before.

An additional parameter considered in this work for the online implementation of the proposed method is the time required to perform all the steps of the method, called latency time. Indeed, mobile windows, as described in Section 4.1, were implemented as follows: the system gets data each second with a sampling frequency of $256 \mathrm{~Hz}$; it performs data processing together with the data collected in the previous second; if a possible absence is detected, the sonification of the signals is made.

In summary, the latency time is given by the sum of the time 
Table 3

Comparison of different classifiers on the same test-set (24 recordings).

\begin{tabular}{|c|c|c|c|c|}
\hline & $\mathrm{F} 1_{\text {score }}$ validation set & BACC & $\mathrm{F} 1_{\text {score }}$ & MCC \\
\hline FGSVM & $93.0 \%$ & $93.0 \% \pm 4.6 \%$ & $89.4 \% \pm 5.8 \%$ & $0.86 \pm 0.07$ \\
\hline $\mathrm{KNN}(\mathrm{k}=1)$ & $89.0 \%$ & $90.0 \% \pm 5.0 \%$ & $85.0 \% \pm 7.0 \%$ & $0.81 \pm 0.08$ \\
\hline Boosted tree & $92.0 \%$ & $92.0 \% \pm 5.0 \%$ & $89.0 \% \pm 6.0 \%$ & $0.86 \pm 0.06$ \\
\hline Logistic regression & $90.0 \%$ & $91.0 \% \pm 5 \%$ & $87.0 \% \pm 6.4 \%$ & $0.83 \pm 0.08$ \\
\hline
\end{tabular}

required to get new data $(1 \mathrm{~s})$ plus the processing time of the method, according to Eq. (1):

$t_{\text {latency }}=t_{\text {acquisition }}+t_{\text {processing }}$

The average processing time on the 24 test cases was found equal to $0.25 \mathrm{~s}$ for each iteration using a Hp Pavillion 15 notebook, i7-5500U processor, CPU $2.40 \mathrm{GHz}$, RAM $16 \mathrm{~Gb}$. Therefore, the average latency time was found to be $1.25 \mathrm{~s}$, that is compatible with a possible online application. This result is in line with the literature of the last few years (Vidyaratne and Iftekharuddin, 2017).

Given the promising results obtained with short-term data, we applied the method to the whole duration $(47 \pm 10 \mathrm{~min})$ of the same testset. The same settings as for the short-term dataset are used (namely, the same filter parameters, temporal and spatial thresholds).

Table 5 shows the results obtained with the FGSVM classifier. The false positive rate parameter (FPR) is added to the evaluation metrics. FPR (Eq. (2)) evaluates the occurrence of mis- classifications in the intercritical phase for long-term recordings:

$F P R=\frac{F P}{F P+T N}$

\section{Discussion}

In this study, we show that the techniques of sonification of EEG signals may have a role in neurological clinical practice, speeding up and facilitating the automatic extraction and interpretation of relevant information, provided that the EEG signal is repeatable and easily identifiable. However, the sonification techniques must be upstream supported by suitable pre-processing steps.

In the literature, EEG signal sonification techniques were proposed as stand-alone techniques (i.e. applied on the whole signal) for the discrimination of critical activities (Väljamäe, et al., 2013). However, for online monitoring activities, this might imply listening to hours of recordings with no useful information content, and thus an overload of information for the clinician not easy to interpret. On the contrary sonification should be aimed at simplifying and reducing the information contained in the EEG signal.

To this aim, the sonification approach proposed in our work could be a valid support for the clinician and potentially also for auxiliary personnel, as the provided sound information can be quickly interpreted as compared to the EEG path. However, this cannot be achieved without an effective pre-processing of the signal to significantly reduce the potentially relevant elements to be acoustically analyzed.

Therefore, a specific filtering technique was set up for the present application. The proposed method, based on the SWT, proved to be a good solution. Critical steps concern the choice of the appropriate wavelet, the number of decomposition levels and of the information reference signal (and relative thresholds). The best wavelet turned out to be a member of the biorthogonal family (Bior 3.3, Table 2).

Spatial threshold was based on physiological considerations of generalized seizures: an absence must concurrently occur on a minimum number of channels that in our case was set as at least half plus one of the EEG channels considered. This choice is a compromise since even if absences are generalized epileptic seizures, they might not start simultaneously in all the channels.

The proposed filtering method does not require an artefact reference channel (like EOG, EMG, ECG), allows the extraction of information of interest only and has a high computational speed (1.25 s average latency time). Table 2 shows that the use of a filtering system based on the source decomposition increases the performance of the subsequent classification phase (increase of about $30 \%$ on BACC). The validation of the proposed filtering method is among the future developments of the method.

Concerning the automatic classification step, the combination of entropy and coherence indices has proved to be suitable for the discrimination of generalized seizures from possible artefacts.

In this study, the average performance was defined on all the EEG channels of each patient under examination. This allowed defining the post-processing phase, which refined and improved the results obtained with the automatic recognition through spatial and temporal thresholds.

In this work, a FGSVM was used as classifier, but the choice of the Boosted Tree can also be suitable. As shown in Table 3 the results obtained are comparable (average $\mathrm{F} 1_{\text {score }}$ on test set $89 \%$ ). Therefore, both the SVM family and the Gradient Boosting algorithms could be compatible solutions for the case under consideration.

Results presented in Table 4 show that, after the application of the online and offline method thresholds (as described in Section 4.3), better performances are obtained with respect to those without any post-processing (FGSVM), with an average increase in the performance parameters of $3.5 \%$ for the BACC, about $5.5 \%$ for the $\mathrm{F} 1_{\text {score }}$ and about 0.08 points for the MCC. The main difference between the offline and online method concerns the variability of the results that could be related to the definition of time threshold for the offline application of the method (see Section 4.3.2): an error propagation might occur if the first few seconds of the seizure are not correctly classified. If not, the OFFLINE performance is slightly better than the ONLINE one, as shown by the $\mathrm{F} 1_{\text {score }}$ value in Table 4.

Our results confirm the possible online use of the method in videoEEG procedures, given that $96 \%$ of balanced accuracy was achieved (Table 4 - ONLINE performance) with an average latency time of only $1.25 \mathrm{~s}$. The performance increased thanks to the proposed filtering procedure and to the choice of the appropriate wavelet.

Regarding long-term data, Table 5 highlights the promising performances of the method even without a specific optimization

Table 4

Comparison of SVM classification with on-line and off-line classification. The test set is made of 24 recordings.

\begin{tabular}{lllll}
\hline & Time duration $[\mathrm{s}]$ & BACC & F1 score & MCC \\
\hline FGSVM (Bior 3.3) & $53 \pm 18$ & $93.0 \% \pm 4.6 \%$ & $89.4 \% \pm 5.8 \%$ & $0.86 \pm 0.07$ \\
ONLINE & $53 \pm 18$ & $96.7 \% \pm 3.9 \%$ & $94.9 \% \pm 4.9 \%$ & $0.94 \pm 0.06$ \\
OFFLINE & $53 \pm 18$ & $96.4 \% \pm 5.8 \%$ & $95.2 \% \pm 7.7 \%$ & $0.94 \pm 0.10$
\end{tabular}


Table 5

Comparison of SVM classification with on-line and off-line classification. The test set is made of 24 recordings whose duration is $47 \pm 10$ min.

\begin{tabular}{|c|c|c|c|c|c|}
\hline & Time duration [minutes] & BACC & $\mathrm{F} 1_{\text {score }}$ & MCC & FPR \\
\hline FGSVM (Bior 3.3) & $47 \pm 10$ & $87.0 \% \pm 6.0 \%$ & $53.0 \% \pm 16.0 \%$ & $0.55 \pm 0.14$ & $2.4 \% \pm 2.0 \%$ \\
\hline ONLINE & $47 \pm 10$ & $89.0 \% \pm 6.0 \%$ & $69.0 \% \pm 15.0 \%$ & $0.70 \pm 0.14$ & $1.1 \% \pm 1.0 \%$ \\
\hline OFFLINE & $47 \pm 10$ & $91.0 \% \pm 5.0 \%$ & $78.0 \% \pm 15.0 \%$ & $0.78 \pm 0.14$ & $0.8 \% \pm 1.0 \%$ \\
\hline
\end{tabular}

procedure. Thanks to the high specificity of the method (about $98 \%$ on average for the three methods), a low FPR is obtained. As shown in Table 5, the BACC parameter (Eq. (13)) does not vary much between short-term and long-term analysis, while the $\mathrm{F} 1_{\text {score }}$ decreases. The PRECISION parameter in the $F 1_{\text {score }}$ formula (Eq. (12)) drastically drops due to the heavy imbalance in the number of test samples between seizures and interictal activity, which inevitably raises the number of false positives (also in case of high values of specificity, 98\% average in our study). On the contrary, with the BACC parameter the unbalance has less influence on the test result because false positives are related only to true negatives. Possible improvements could be obtained adding more reference signals and finding other features for discrimination. These features and problems will be taken into account in future developments.

We observed the sonification of the seizures to be slightly delayed since we considered that absences lasting less than two seconds do not allow the clinician focusing on the patient and related clinical signs. At the same time, we have chosen to sonify the first two seconds with two pre-alarms in order not to miss the first meaningful time instants of possible events of interest setting up a pre-alert.

In other words, if a perfect machine learning algorithm (i.e. $100 \%$ accuracy) were implemented, the sonification would highlight only the true critical events and therefore would only function (however already useful) of automatic alert for the clinician. As an algorithm with such performance for online EEG monitoring on children is not readily achievable, the sonification would also provide support for non-critical events detected as critical. For example, any false positives with a duration of less than three seconds would be discriminated against a real critical activity (since in the first case only the two pre-alarm beeps would sound).

To better explain the usefulness of the sonification techniques applied to EEG signals, Fig. 1 shows an example of application of the proposed method to a long-term recording (time duration of about $2500 \mathrm{~s}$ ). The epileptic seizure is marked with a dashed rectangle. Above: one EEG channel is shown; below: the corresponding filtered and sonified signal. Some false positives appear, most $<2 \mathrm{~s}$ of duration. The cases marked with continuous rectangle have longer duration. However, these cases can be perceptually discriminated because the amplitude of the sound signal is lower than that perceived during the seizure.

Thus, though at a qualitative level, the method allows a considerable reduction of the information to be analyzed by the clinician. Moreover, the sound information can be used for the evaluation of the beginning of the seizure in online activities, giving to the clinician an immediate feedback on the patient's state of health, without requiring to watch video-EEG on the monitor and thus focusing on the patient to evaluate the main clinical signs, such as the degree of consciousness in the case of epileptic absences. In addition, when the audio alarm stops, the clinician could have an almost instantaneous feedback about the end of the seizure.

The presented method has some limitations: the impossibility of discriminating false negatives, detecting only false positives; the sound information, if used as the only discriminating factor, can be highly subjective but, according to Bonebright et al. (2011) it will be a possible future development.

Furthermore, combining a specific sound to a given event would allow possible future applications using other sounds to recognize different characteristic events in the EEG channels (such as IED).

No statistical evaluation was carried out about the possibility of discriminating both at a perceptive level and through objective indices (by the sonification of the d6 level coefficients) possible artefacts and epileptic seizures, as to date in the authors' knowledge there is not a method generally recognized as reliable and repeatable (Väljamäe, et al., 2013). Indeed, the only methodology currently recognized for
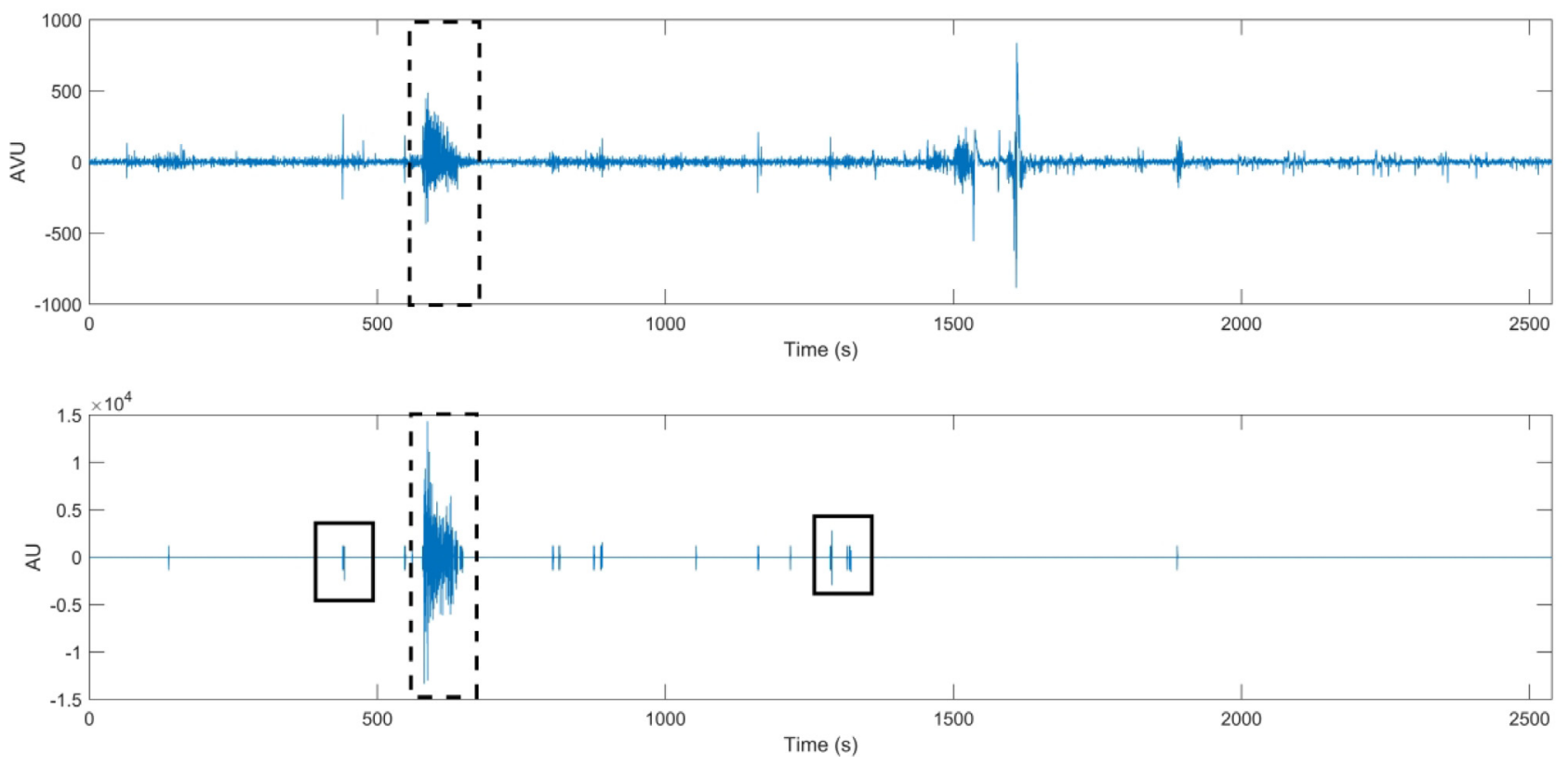

Fig. 1. Example of sonification. Above: an EEG channel; below: the sonified signal. 
auditory display evaluation is the one described by Bonebright et al. (2011). At present, the method used to evaluate accuracy consists in defining a group of subjects for listening and selecting the audio tracks to be discriminated (double blind test). This approach was not an aim of this paper, but certainly represents a potential future development.

\subsection{Conclusions}

We explored the possibility of finding a compromise between computational speed and accuracy for a fast and reliable identification of nonmotor generalized onset seizures. To this aim, we propose a specific filtering technique for the clinical problem under examination, compatible with its online implementation. In addition, we developed an appropriate alert technique that requires low latency time to inform the clinician about seizure onset. Our results, though preliminary, suggest a possible optimization of existing methods applied to specific clinical settings.

From the encouraging results obtained on the available dataset we believe that, after the validation and optimization steps, the method could also be successfully applied to long-term data.

In this framework, we chose absences as an 'optimal model' to test our methodology, being aware that owing to their electroclinical characteristics, they are easily recognised and processed for sonification. However, the good results obtained, both in terms of balanced accuracy (about 96\%) and latency times (1.25 s on average), offer the perspective for a real-time application of our methodology in the clinical setting of other types of seizures, which are more challenging for automatic recognition and online sonification. For instance, atypical absences, which typically occur in the context of severe epilepsies like the Lennox-Gastaut syndrome, and are often associated with traumatic falls, make it worth implementing a methodology of automatic recognition. In this study, the accuracy of our method does not seem to be affected by the electroclinical characteristics of atypical absences i.e. less abrupt onset or termination and generalized slow spike and wave discharges on the EEG. However, the small sample prevents us to draw definite conclusions on this point.

Our methodology might also be helpful during video-EEG monitoring of candidates to epilepsy surgery, which requires a rapid intervention of the clinical staff at seizure onset and the real time evaluation of the related clinical signs, in order to better identify the seizure onset zone. In addition, our method might support clinicians in the interpretation of ictal video-EEG recordings through automatic event recognition techniques and the reduction from 19 simultaneous paths to a single sound information, i.e. a predefined range of sounds combined with each characteristic event found in EEG data (e.g., seizure or interictal epileptiform discharges).

A fast-online sound feedback of the characteristic events (ictal or interictal activities) of the EEG signals could also be applied to get an immediate feedback of seizures in at risk neonates in neonatal intensive care units (NICU) and thus provide their best management (Temko et al., 2017). This approach could also help less experienced users with multi-channel EEG data, especially with $24 / 7$ monitoring in NICU. However, neonatal seizures are much more variable in their clinical presentation and ictal onset pattern on EEG compared to absences. Another limit can be represented by the definition of only one possible information reference signal. Most likely, it will be necessary to first investigate the spectrum of neonatal seizures in search of a series of signals at least representative of a large part of the population under observation. Further longitudinal studies are needed in order to implement the methodology and reduce, for instance, muscle artefacts and seizure onset variability.

A further aspect to be taken into consideration is the number of wavelet decomposition levels, that was defined on a physiological basis for the absence seizures. Its effectiveness should be verified for other types of seizures. Moreover, the features described in this study may not be sufficient to discriminate some types of seizures, such as those with predominant slow wave activity.

Finally, we point out that we are presenting here a method for automatic detection and sonification of a specific type of seizures and its potential practical applications. Cinical trials and related operational performances will be addressed in future works.

\section{Methods and material}

Considering the aims and problems mentioned in Section 1, we propose a method made up of three steps: filtering, automatic recognition and post-processing.

The proposed methods are implemented under MATLAB software (version 2017b) installed on Hp Pavillion 15 notebook (OS Windows 10, 64 bit) Intel Core i7-5500U processor, CPU $2.40 \mathrm{GHz}, \mathrm{RAM} 16 \mathrm{~Gb}$.

To reduce artefacts, the first step consists in pre-processing of the EEG signal with the Stationary Wavelet Transform (SWT) filtering. As compared to the classical Discrete Wavelet Transform (DWT), the SWT has the advantage of being shift-invariant, thanks to the up-sampling operation of the filter coefficients (Guo et al., 1996).

The second step consists in the automatic detection of the epileptic seizures using Fine Gaussian Support Vector Machine (FGSVM).

Finally, the third step performs a post-processing of the results of the classification that allows data processing in real-time through a sonification technique.

The overall procedure is depicted in Fig. 2.

\subsection{Denoising with SWT}

In this section the filtering method is presented. Firstly, the filter design criteria for a correct online application will be defined. In each iteration of the method an information reference signal of epileptic absences will be compared to the portions of the signal under examination. Then, the wavelet used for the SWT operation will be described, along with the selection of the decomposition levels. Finally, the filtering technique will be described: for each level of decomposition we applied a garrote shrinkage function with modified threshold,

\section{Denoising with SWT (bior 3.3)}

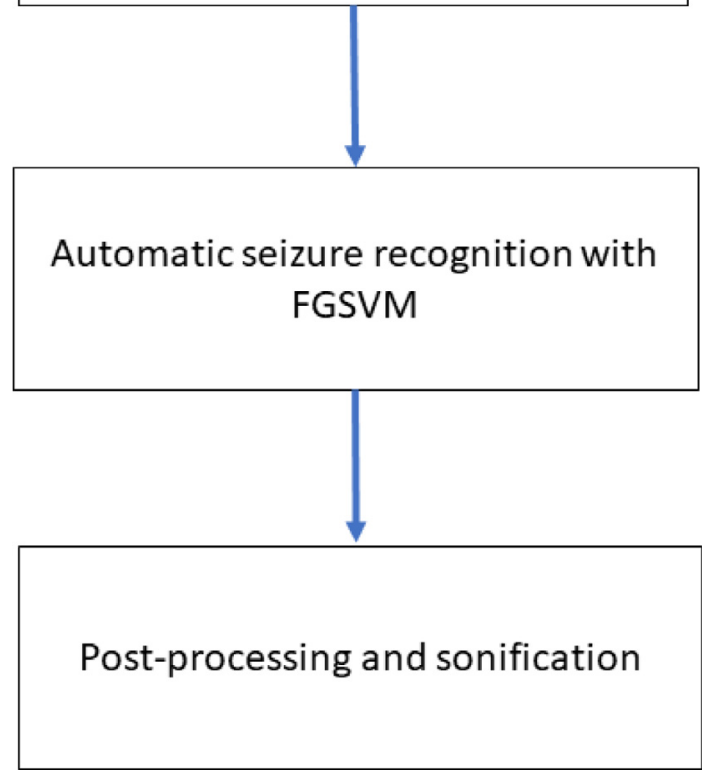

Fig. 2. Workflow of the proposed method. 


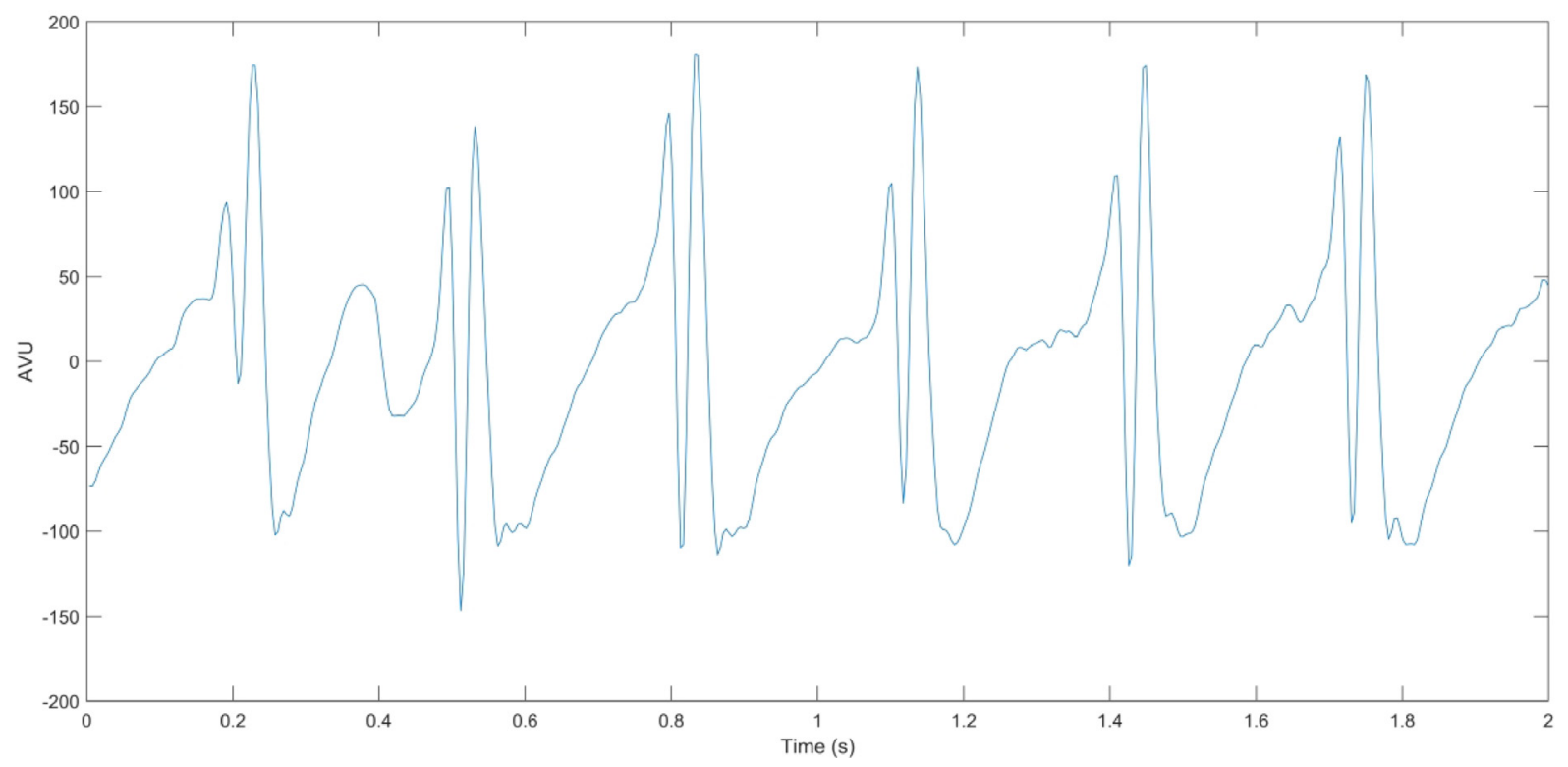

Fig. 3. Example of Information Reference Signal.

based on the degree of correlation between the signal under examination and the information reference signal.

The filtering method proposed here was implemented according to the following requirements (Zhu, 2008; Patel, 2014):

- possible online implementation, that requires a low computational time;

- only EEG signals are considered for artefacts detection;

- method optimized for a specific clinical case: epileptic absences (given the presence of only one type of information reference signal).

Therefore, according to Md Kafiul Islam et al. (2016), the filtering method consists of a number of steps.

The first step is the selection of an EEG signal consisting in an absence seizure which will represent our information reference signal. To this aim, a signal of length equal to $2 \mathrm{~s}$ out of the database was selected, indicated by an expert. An example is shown in Fig. 3. It will be compared to the EEG signal under examination.

At each iteration and for each EEG channels moving windows of $2 \mathrm{~s}$ of duration are extracted from the original signals. An example (channel C4-P4) is reported in Fig. 4. Time windows consist of 512 samples at $256 \mathrm{~Hz}$ sampling frequency, with one epoch overlap (i.e. $50 \%)$.

In addition to the reference signal (Fig. 3), the SWT decomposition step is applied to each window under examination. This is indeed a crucial step in the filtering method. The choice of the mother wavelet, if not appropriate, can significantly alter the result of the decomposition.

Taking this into account and according to Upadhyay (2016), in the present work the biorthogonal wavelet family was selected that gave an increased performance as compared to the Haar one proposed in $\mathrm{Md}$ Kafiul Islam et al. (2016) (Table 2). Specifically, we choose the biorthogonal 3.3 wavelet with a different level of decomposition (6 instead of 8).

The level 6 of decomposition (d6) was chosen, both according to physiological considerations and because a further level of decomposition would have included unreliable coefficients. Indeed, the level d6 approximately concerns the frequency range between $2 \mathrm{~Hz}$ and $4 \mathrm{~Hz}$ where the frequencies typical of an epileptic absence seizure are included (epilepsydiagnosis, 2017). An example is shown in Fig. 5.

A first consideration concerns levels $\mathrm{d} 1$ and $\mathrm{d} 2$ : as they do not carry useful information to the present application, their coefficients are set to 0 . Similarly, for the a6 coefficients of approximation: although containing useful information, they concern the level of decomposition with the worst signal-to-noise ratio.

In fact, these levels might include a part of the information of the delta wave and of the low frequency artefacts, such as slow movement artefacts, that, given their high contribution in terms of energy, significantly alter the signal trend (Md Kafiul Islam et al., 2016).

Since the main contribution of absences is above $2 \mathrm{~Hz}$, the information between $0 \mathrm{~Hz}$ and $2 \mathrm{~Hz}$ is considered unnecessary for this application. Moreover, the signals were already pre-filtered in the range $1.6 \mathrm{~Hz}-32 \mathrm{~Hz}$ (further details in Section 2). Therefore, the coefficients of this level were set to 0 . Taking these aspects into account, only the contribution represented by the frequency range between $1.6 \mathrm{~Hz}$ and $2 \mathrm{~Hz}$ is disregarded.

The remaining decomposition levels from $\mathrm{d} 3$ to $\mathrm{d} 6$ are compared to the corresponding ones of the information reference signal through the correlation index:

$\rho(\mathrm{A}, \mathrm{B})=\frac{1}{\mathrm{~N}-1} \sum_{\mathrm{i}=1}^{\mathrm{N}}\left(\frac{\overline{\mathrm{A}_{\mathrm{i}}-\mu_{\mathrm{A}}}}{\sigma_{\mathrm{A}}}\right)\left(\frac{\mathrm{B}_{\mathrm{i}}-\mu_{\mathrm{B}}}{\sigma_{\mathrm{B}}}\right)$

The index $\mathrm{C}_{\mathrm{s}}=|\rho(\mathrm{A}, \mathrm{B})|$ that varies in the range (Swisher, 2017) is a measure of similarity between the signal (A) and the reference (B) for a given level (Aarabi et al., 2008).

Thus, for coefficients $\mathrm{d} 3$ to $\mathrm{d} 6$, the garrote shrinkage function (Gao, 1998) was chosen as filtering method according to Eq. (4). The coefficients $d_{i, j}$ of the $j$-th decomposition level are filtered if they are greater than a given threshold $t_{i, j}$ that varies at each level of decomposition.

$g(i, j)=\left\{\begin{array}{l}d_{i, j}\left|d_{i, j}\right| \leq t_{i, j} \\ \frac{t_{i, j}}{d_{i, j}}\left|d_{i, j}\right|>t_{i, j}\end{array}\right.$

ti, $\mathrm{j}$ is the modified universal threshold (Md Kafiul Islam et al., 2016) defined as:

$t_{i, j}=K \alpha_{i, j} \sqrt{2 \ln N}$

where $\alpha_{i, j}$ is defined as:

$\alpha_{i, j}=\frac{\text { median }\left(\left|w_{i, j}\right|\right)}{0.6745}$

And $\mathrm{w}_{\mathrm{i}, \mathrm{j}}$ is the value of the coefficients at the considered level.

The value of $\mathrm{K}$ in Eq. (5) varies according to the correlation index $\mathrm{C}_{\mathrm{s}}$, 


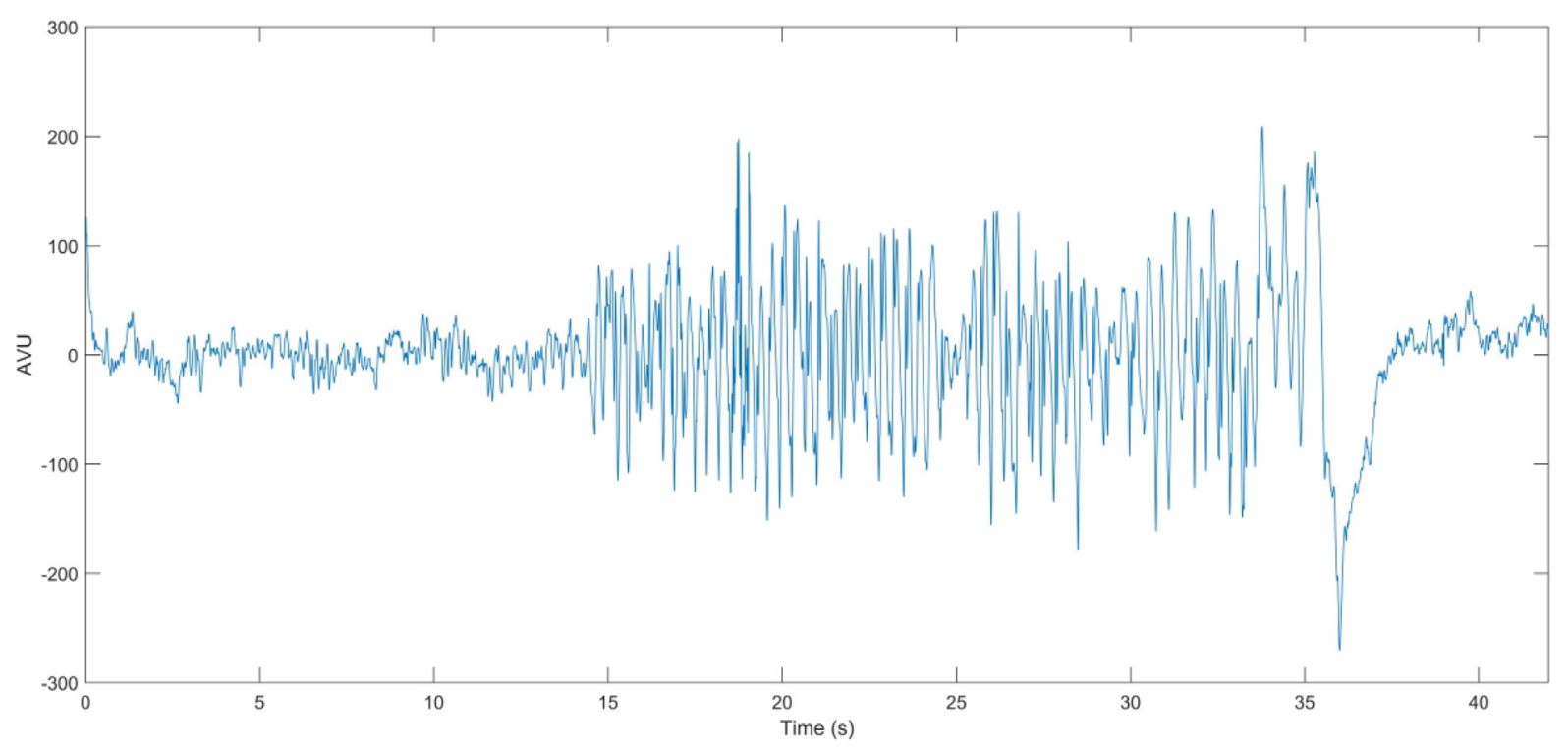

Fig. 4. Example - Raw EEG channel C4-P4.

according to the following decision process:

1. If $C_{s} \geq T_{\text {high }} \rightarrow$ nodenoising

2. If $T_{\text {low }} \leq C_{S}<T_{\text {high }} \rightarrow K=1.5$

3. Otherwise $\mathrm{K}=1$

The thresholds $\mathrm{T}_{\text {high }}=0.7$ and $\mathrm{T}_{\text {low }}=0.5$ were chosen empirically: they determine when the signal has a high or a low level of similarity with the information reference signal and thus apply a tunable filtering by varying the index $\mathrm{K}$.

When each level of decomposition has been analyzed, the signal can be reconstructed applying the Inverse Stationary Wavelet Transform (ISWT). As an example, Fig. 6 shows how the contribution of the artefact localized at the end of the seizure $(35 \mathrm{~s}-40 \mathrm{~s}$, marked with a dashed circle) is considerably attenuated by the proposed method.

For this filtering method the validation step (for example the choice of the best setting for the thresholds $\mathrm{T}_{\text {low }}$ and $\mathrm{T}_{\text {high }}$ or the best information reference signal) was not performed, as it was not the aim of the paper. However, given the good results obtained (see Section 2 devoted to results and Fig. 7), future developments of the method will certainly concern its validation.

To highlight the good performance of the proposed filtering method, in Fig. 7 other results are reported, each signal belonging to a different patient. On the left the original signals are shown, and on the right the corresponding filtered signals.

\subsection{Automatic recognition by SVM}

The next step of the method concerns the search of features capable to detect an epileptic absence in the whole EEG signal, that is, discriminating an ictal phase from an interictal phase.

In the literature, the field of useful features for seizure detection is almost boundless and has been widely discussed in recent years (Upadhyay, 2016; Boubchir, 2017). In this work we focus both on the detection of features from the time series and on those related to the source decomposition. The aim is that of evaluating only the features

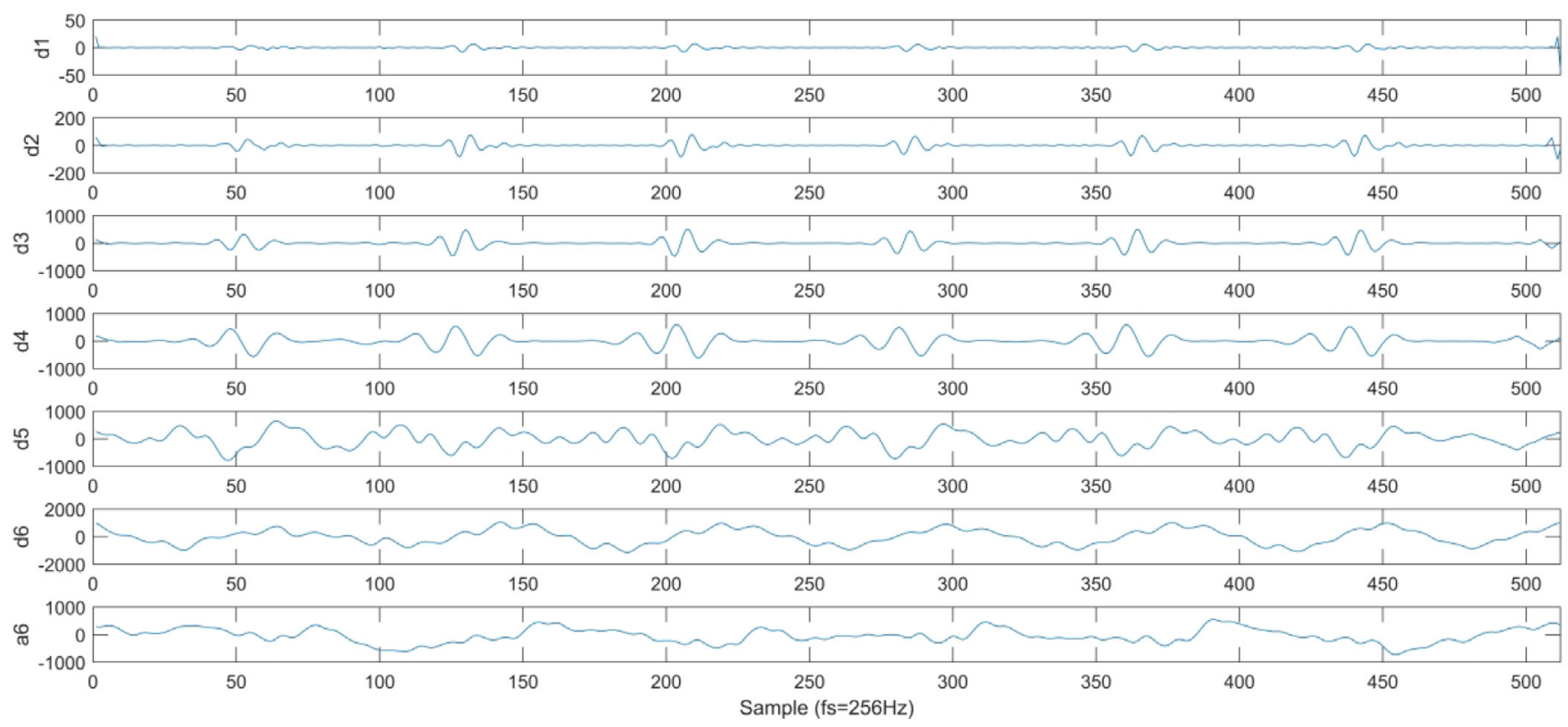

Fig. 5. Decomposition with SWT (6-levels). 

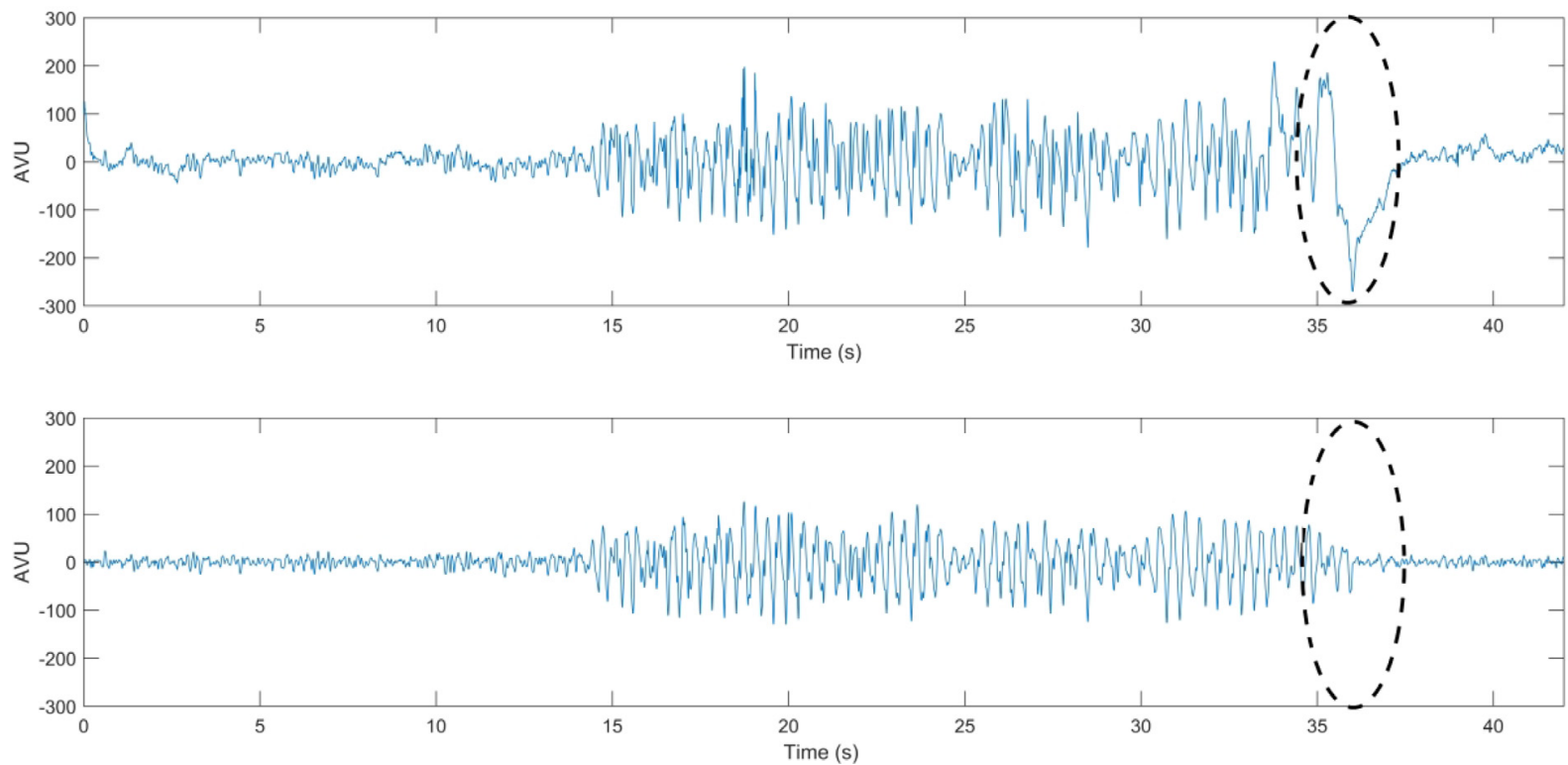

Fig. 6. Example of successful filtering: the artefact occurring around $35 \mathrm{~s}-40 \mathrm{~s}$ is filtered out.
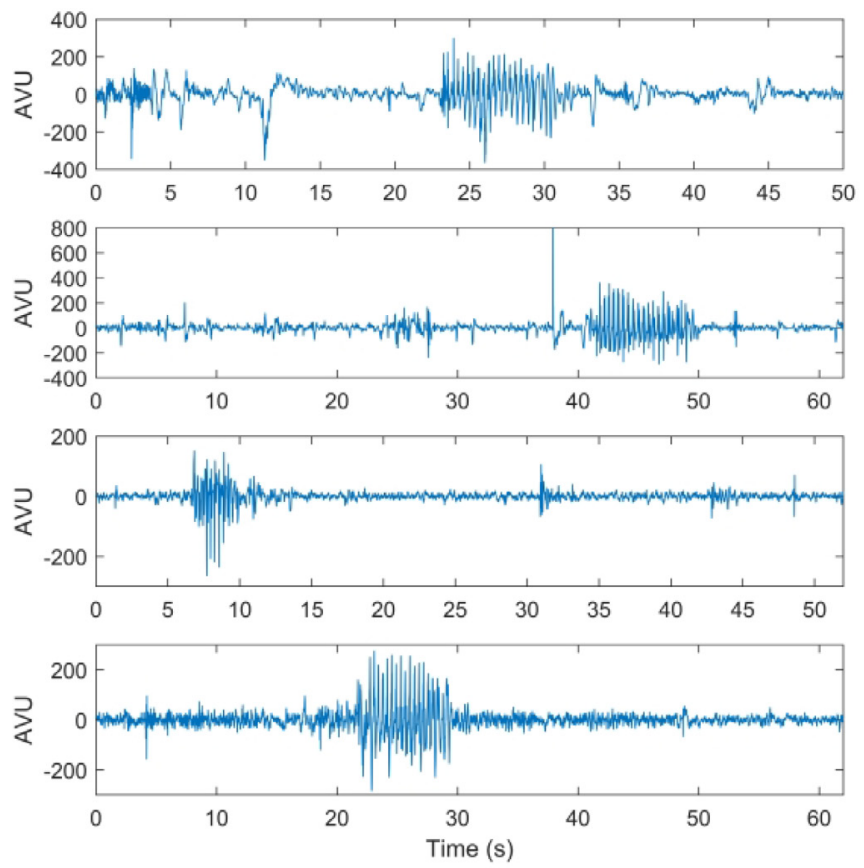
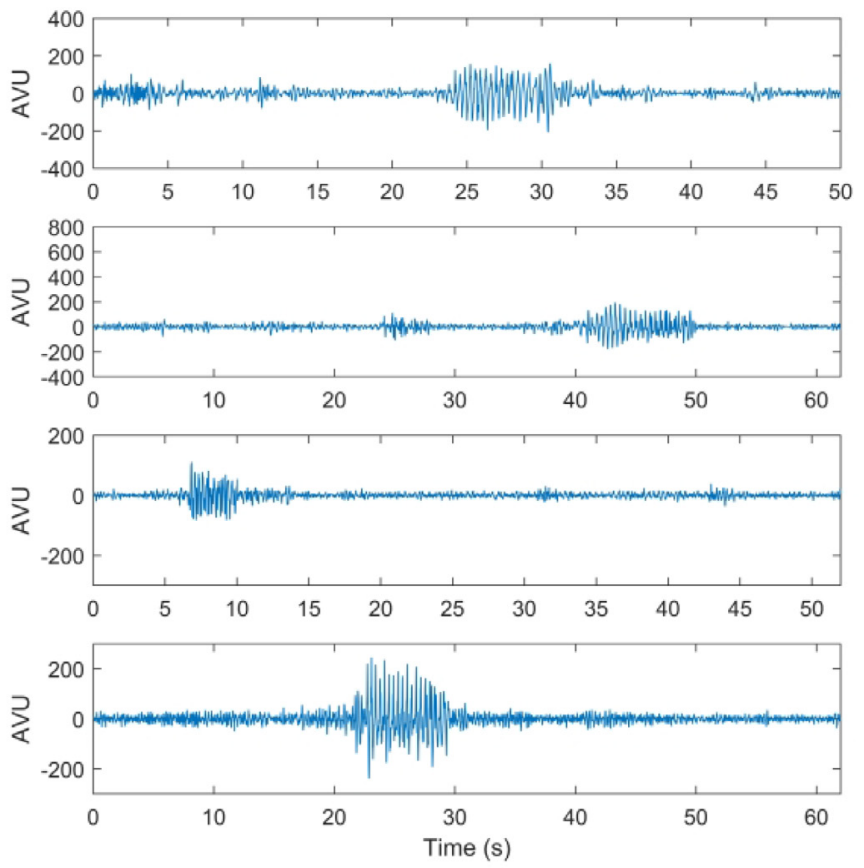

Fig. 7. Other Example of filtering. Left original signals; right: filtered signals.

allowing the online application of the proposed method, which therefore must not require burdensome processing time.

Therefore, in the following sections only the features considered for the classification step will be described.

\subsubsection{Permutation Entropy}

Following the definition given by Bandt (2002), the calculation of the Permutation Entropy for a given time series $\times$ (of length $N$ ), can be described as follows:

- From the time series build (N-m-1) delay vectors: $X_{t}=\left[x_{t}, x_{t+\tau}, \ldots, x_{t+(m-1) \tau}\right]$ with $t=0:(N-m-1)$, where $m$ is the embedding dimension and $\tau$ the time delay.

- Each vector is rearranged in ascending order $X_{t_{\text {reord }}}=\left[x_{t+\left(j_{1}-1\right) \tau} \leq x_{t+\left(j_{2}-1\right) \tau} \leq \ldots \leq x_{t+\left(j_{d}-1\right) \tau}\right]$, where $\mathrm{j}$ is the positional shift of the original element.

- For each delay vector there will be $m$ ! reorder patterns, $\pi_{i}, i=1 \ldots$ $\mathrm{m}$ !, also called "motifs". As an example, for $\mathrm{m}=3$ there are 6 possible motifs.

The new positional order of the elements in the vector $X_{t_{\text {reord }}}$ as compared to the elements of $X_{t}$, defines which pattern has been obtained for a given delay vector. Thus, for each delay vector which reordering pattern has been applied to its elements can be evaluated, comparing it with the reordered vector.

The possible occurrences of the $\pi \mathrm{j}$-th motif in the time series are indicated with $f\left(\pi_{j}\right)$. The relative frequency $p\left(\pi_{j}\right)$ is given by Eq. (7).

$p\left(\pi_{j}\right)=\frac{f\left(\pi_{j}\right)}{N(-m-1) \tau}$ 


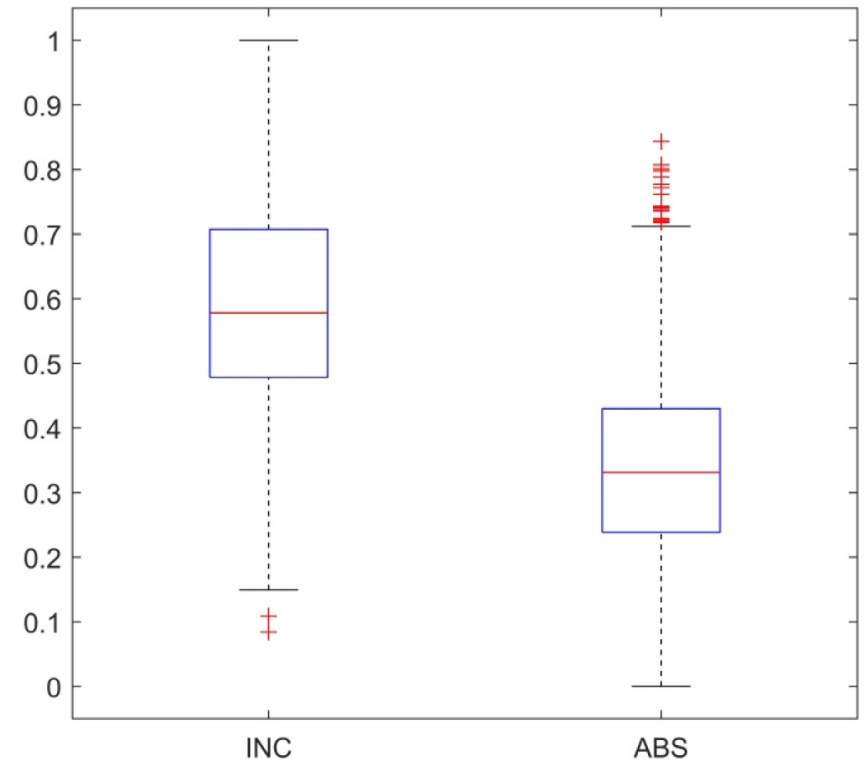

Fig. 8. Permutation Entropy box-plot for the two classes considered: absence (ABS) and interictal (INC).

According to Bandt (2002), in this work $\mathrm{m}=3, \tau=1$, and $\mathrm{N}=512$.

It is thus possible to analytically define the Permutation Entropy through the following Eq. (8):

PermEntropy : $=-\sum_{\pi_{j}}^{m !} p\left(\pi_{j}\right) \log _{2} p\left(\pi_{j}\right)$

Fig. 8 shows the box-plot of the Permutation Entropy's features of the two classes used in the training set of this work: absence (ABS) and interictal (INC).

However, in some cases we found that the entropic index alone was not enough meaningful for a correct identification of the seizure. Therefore, other features were added for classification that will be described in the following sections. This allowed obtaining a balanced accuracy of about $96 \%$ in the online modality. The performance was found comparable to the offline mode.

\subsubsection{Kurtosis}

Kurtosis quantifies whether the tails of the data distribution matches the Gaussian distribution. Specifically, the Kurtosis of the decomposition levels $\mathrm{d} 3$ and $\mathrm{d} 4$ were considered.

As described in (Upadhyay, 2016; Lofhede, 2010) the kurtosis index of such frequency ranges is often used as a statistical feature in the classification methods concerning EEG signals. It is defined as follows:

Kurtosis : $=\frac{\sum_{i=1}^{n}\left(x_{i}-\bar{x}\right)^{4}}{(n-1) \sigma_{x}^{4}}$

In Figs. 9 and 10 the box-plots of the Kurtosis's features for the decomposition level $\mathrm{d} 3$ and $\mathrm{d} 4$ used in the training set of this work are presented. For these decomposition levels a noticeably difference is shown between the interictal phase and those related to the absences. This difference was not so evident in the other levels of decomposition, considering also the values obtained through the calculation of the Fisher Score (FS) (Upadhyay, 2016) on the features of the training set, where the following values were obtained: $\mathrm{FS}_{\text {Kurtosis-d3 }}=1.40$; $\mathrm{FS}_{\text {Kurtosis-d } 4}=0.86 ; \mathrm{FS}_{\text {Kurtosis-d5 }}=0.05 ; \mathrm{FS}_{\text {Kurtosis-d6 }}=0.41$. Therefore, the corresponding values of Kurtosis (levels d5 and d6) were not considered.

\subsubsection{Index of coherence}

The index of coherence gives a measure of phase synchronization

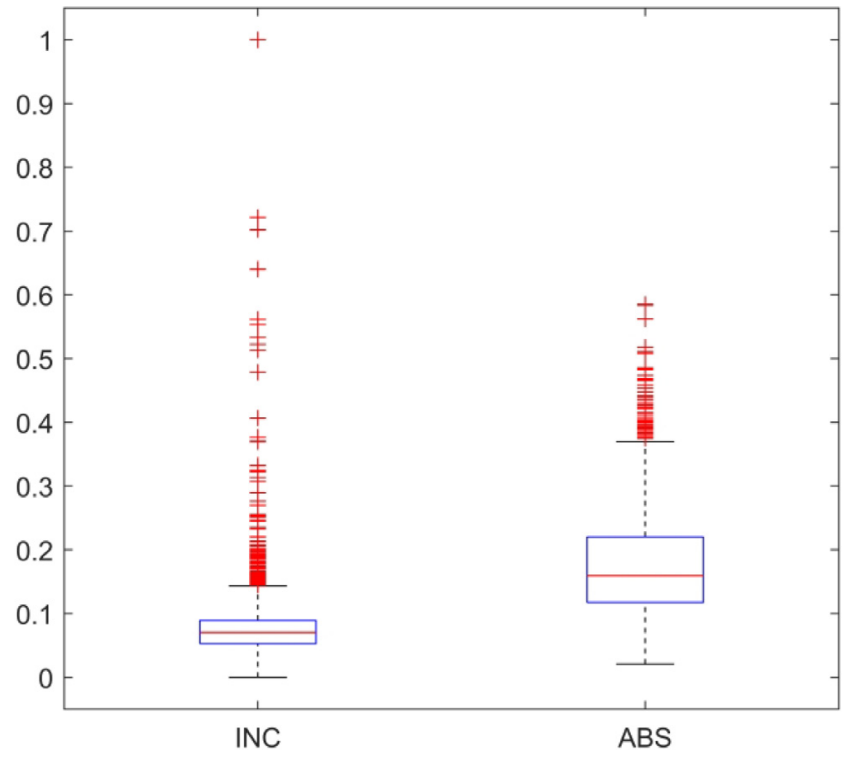

Fig. 9. Decomposition level d3: Kurtosis box-plot for the two classes considered: absence (ABS) and interictal (INC).

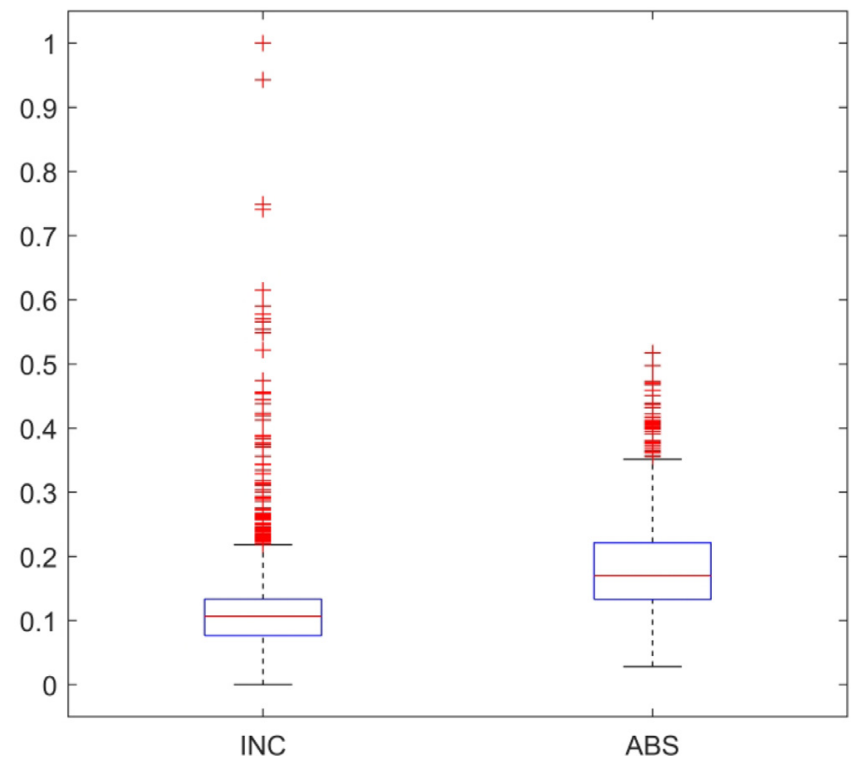

Fig. 10. Decomposition level d4: Kurtosis box-plot for the two classes considered: absence (ABS) and interictal (INC).

between EEG signals. In Mormann (2000) it was shown that it can detect interictal periods among ictal periods, according to their degree of synchronization.

The Mean Magnitude-squared coherence (Mormann, 2000) was used here as a possible index of coherence:

$C_{x y}(f)=\frac{\left|P_{x y}(f)\right|^{2}}{P_{x x}(f) P_{y y}(f)}$

where $P_{x y}(f)$ represents the cross power spectral density between two derivations ( $\mathrm{x}$ and $\mathrm{y}$ ) calculated for each frequency, while $P_{x x}(f)$ and $P_{y y}(f)$ are the power spectral densities of signal $\mathrm{x}$ and $\mathrm{y}$, respectively.

In this work, only the coherence values included in the frequency range typical of absences (i.e. $2 \mathrm{~Hz}-4 \mathrm{~Hz}$ ) were considered.

Fig. 11 shows the box-plot of the Coherence features used in the training set of this work.

The figure shows the values (range [01]) of coherence for the elements of the training set labelled as interictal and those defined as 


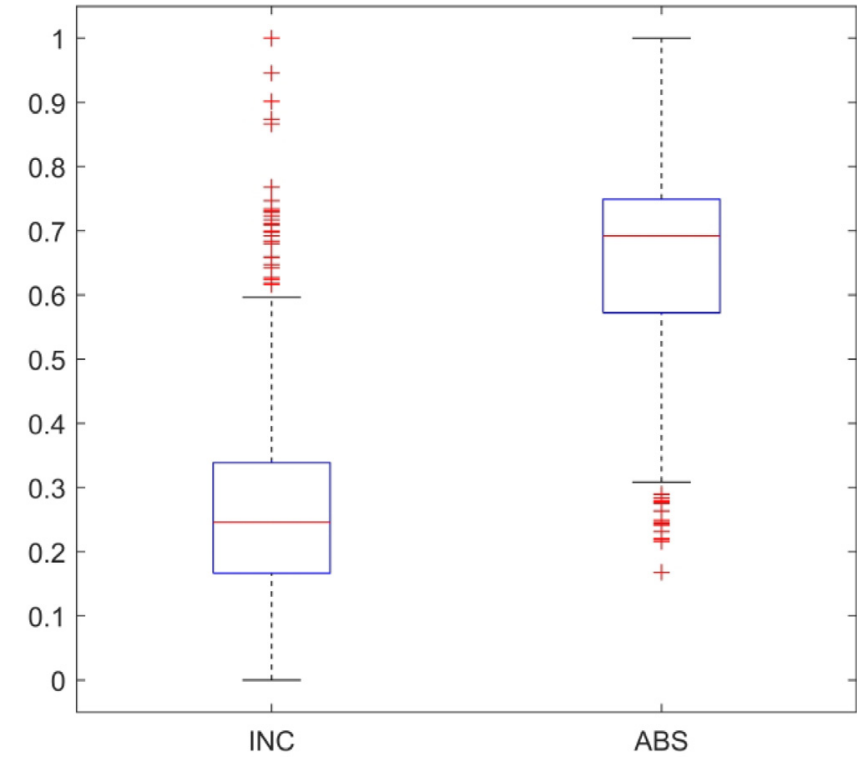

Fig. 11. Coherence box-plot for the two classes considered: absence (ABS) and interictal (INC).

absences.

\subsubsection{Choice of the classifier}

After the filtering process with SWT, the features described above are calculated for each time window and each EEG channel.

The analysis now focuses on the features that will set up the training set. Before the classification step preliminary operations are carried out on these features.

First, possible outliers are detected. The method chosen for evaluation and subsequent removal of outliers is the following: if the data under examination $\mathrm{x}_{\mathrm{i}} \in \mathrm{X}$ (with $\mathrm{X}=\left[\mathrm{x}_{1} \ldots \mathrm{x}_{\mathrm{n}}\right]$ elements of the training set) is three times larger than the absolute deviation of the median MAD (MAD : $=\mathrm{K}^{*}$ median $\left(\left|\mathrm{x}_{\mathrm{i}}-\operatorname{median}(\mathrm{X})\right|\right)$ with $\mathrm{K}=1.4826$ (Sachs, 1984), it is recognized as an outlier and eliminated.

Subsequently, the remaining features are normalized. To this aim, the data are normalized in the interval (Swisher, 2017) according to Eq. (11):

$x_{i_{\text {norm }}}=\frac{x_{i}-\min (X)}{\max (X)-\min (X)}$

For the choice of the possible classifier, the MATLAB tool (version 2017b) "Classification Learner" (MATLAB and Statistics and Machine Learning Toolbox Release, 2017b) was used.

As a first criterion for the choice of the optimal classifier for the test phase, only the classifiers with the highest $\mathrm{F} 1_{\text {score }}$ obtained during the validation phase of the training set via $\mathrm{k}$-fold-cross-validation $(\mathrm{k}=10)$ were considered. $\mathrm{F} 1_{\text {score }}$ is defined as:

$F 1_{\text {score }}=2 \frac{\text { PRECISION } \times \text { RECALL }}{\text { PRECISION }+ \text { RECALL }}$

Following the operations described above, the best classifier identified by the Classification Learner was found to be of the Support Vector Machine family (SVM) (Burges, 1998), specifically a Fine Gaussian SVM.

For the test phase of the possible classifiers the following performance indices (in addition of $\mathrm{F} 1_{\text {score }}$ ), optimal for unbalanced datasets, were used:

- Balanced Accuracy (BACC)
$\mathrm{BACC}:=\frac{\frac{T P}{T P+F N}+\frac{T N}{T N+F P}}{2}$

- Matthews Correlation Coefficient (MCC)

$\mathrm{MCC}:=\frac{T P \times T N-F P \times F N}{\sqrt{(T P+F P)(T P+F N)(T N+F P)(T N+F N)}}$

Since the method was tested on a data set made of recordings of quite short time duration, no evaluation of the false positive rate parameter was performed. It will be taken into account in future developments of the method, specifically in the clinical validation of the method on long-term data.

\subsection{Post-processing and sonification}

The last part of the methodology consists in applying spatial and temporal thresholds to the results obtained from the automatic recognition.

Spatial threshold was defined through an empirical criterion, based on physiological considerations of generalized seizures: an absence must concurrently occur on a minimum number of traces that in our case it was set as at least half plus one of the EEG channels considered.

As for the temporal threshold, a part of the signal is defined an absence if and only if it is classified as absence for at least two seconds, and separated each other by at most one second (to avoid possible false negatives). This value was chosen based on two considerations: absences with a duration less than two seconds are unlikely to occur; and in those lasting between 2 and $4 \mathrm{~s}$ it is difficult to identify any clinical sign (da Silva, 1987; Keilson, 1987).

\subsubsection{Online method}

For the implementation of the online method only the spatial threshold was used, as the temporal threshold implies time delays not compatible with an almost real-time response during the exam.

Specifically, a minimum number of channels was chosen given by half of them (10 channels in our case).

The sonification procedure works as follows: if the automatic recognition and related post-processing steps detect three (or more) consecutive absences (ABS), the first 2 consecutive seconds will produce 2 beeps $\left(\right.$ beep $_{1}$ and beep $_{2}$ ), which allow a first discrimination between a possible absence and a false positive, since absences of less than two seconds cannot be considered as possible seizures but just as particular interictal activities. They are anyway kept in memory by the method for the offline phase. Then the third one (and following ones) will produce a sound pointing out the epileptic absence, named sound $_{a b s}$.

The online processing for sonification described above is thus given by the following relationship:

$[\mathrm{ABS}-\mathrm{ABS}-\mathrm{ABS}] \Rightarrow\left[\right.$ beep $_{1}$ beep $_{2} \rightarrow$ sound $\left._{a b s}\right]$

This sound is obtained considering only the coefficients attributable to the main frequency range of absences (i.e. $\mathrm{d} 6$ for all the $\mathrm{N}$ channels) and sonified according to Eq. (15):

sound $_{a b s}=\sum_{i=1}^{N} E_{-}$sound $_{i} \sin \left(2 \pi f_{\text {osc }} t\right)$

where E_sound are the coefficients (over sampled to be reproducible at $48 \mathrm{kHz}$, the playback audio frequency) and the sound oscillation frequency $f_{\text {osc }}$ is set to the note $\mathrm{C}$ at $256 \mathrm{~Hz}$. This value was chosen empirically for the sonification phase following the perceptual evaluation during the test phase: lower frequencies did not allow appreciating rapid signal variations, while higher frequencies brought the effect of enhancing small variations of the signal attributable to possible effects of noise on the signal. Similar approaches are reported in (Colostate.edu, 2018). 


\subsubsection{Offline method}

As in usual automatic seizure detection systems, an offline method (i.e. at the end of the EEG exam) was also implemented. The temporal threshold $(2 \mathrm{~s}$ ) has been added to eliminate further false positive cases. Indeed, in our method the temporal threshold can be set by the clinician, thus allowing discarding all those absences with a duration shorter than the chosen time threshold.

The offline method follows the classic clinical indications of what an EEG report should provide. At the end of the EEG examination a summary report is produced in which the following information is reported:

- Number of absences detected, their duration and time location;

- Typical average frequency, to assess any atypical absences;

- Derivation involved and duration of the involvement.

\section{Acknowledgments}

This work was in part carried out under projects 2016.0952 and 2015.0911, funded by Fondazione Ente Cassa Risparmio Firenze, Firenze, Italy.

\section{References}

Aarabi, A., et al., 2008. Does spatiotemporal synchronization of EEG change prior to absence seizures? Brain Res. 1188, 207-221.

Abd Rahman, F., et al.,2015. A review on the current state of artefact removal methods for electroencephalogram signals. IEEE 10th Asian Control Conference (ASCC).

Alam, S.M.S., et al., 2013. Detection of seizure and epilepsy using higher order statistics in the EMD domain. IEEE J. Biomed. Health Inform. 17 (2), 312-318.

Alotaiby, Turkey N, Alshebeili, Saleh A, Alshawi, Tariq, Ahmad, Ishtiaq, Abd El-Samie, Fathi E, 2014. EEG seizure detection and prediction algorithms: a survey. EURASIP J. Adv. Signal Process. 2014 (1)

Bandt, C., et al., 2002. Permutation entropy: a natural complexity measure for time series. Phys. Rev. Lett. 88, 174102-174104.

Bonebright, T.L., 2011. Evaluation of Auditory Display. In: Hermann, Thomas, Hunt, Andy, Neuhoff, John G. (Eds.), The Sonification Handbook. Logos Verlag, Berlin, Germany, pp. 111-141.

Boubchir, L., et al., 2017. A Review of Feature Extraction for EEG Epileptic Seizure Detection and Classification. 40th International Conference on Telecommunications and Signal Processing IEEE, Barcelona, Spain, pp. 456-460.

Boudet, S., et al., 2012. Improvements of adaptive filtering by optimal projection to filter different artefact types on long duration EEG recordings. Comput. Methods Programs Biomed. 108, 234-249.

Burges, C.J.C., 1998. A tutorial on support vector machines for pattern recognition. Data Min. Knowledge Discov. 2, 121-167.

Chavez, M., et al., 2018. Surrogate-based artefact removal from single-channel EEG. IEEE Trans. Neural Systems Rehabilitation Eng. 26 (3).

http://www.cs.colostate.edu/eeg/main/projects/sonification last access: 14/07/2018.

da Silva, L., 1987. Electroencephalography: Basis Principles, Clinical Applications and Related Fields. Urban\&Schwarzenberg.

https://www.epilepsydiagnosis.org/syndrome/cae-overview.html last access: 05/12/ 2017.
Fisher, Robert S., Cross, J.H., French, Jacqueline A., Higurashi, Norimichi, Hirsch, Edouard, Jansen, Floor E., Lagae, Lieven, Moshé, Solomon L., Peltola, Jukka, Roulet Perez, Eliane, Scheffer, Ingrid E., Zuberi, Sameer M., 2017. Operational classification of seizure types by the International League Against Epilepsy: Position Paper of the ILAE Commission for Classification and Terminology. Epilepsia 58 (4), 522-530.

Guo, H., et al., 1996. Convolution using the undecimated discrete wavelet transform. Proc. IEEE Int. Conf. Acoust. Speech Signal Process. 3, 1291-1294.

Hamaneh, M.B., et al., 2014. Automated removal of EKG Artefact from EEG data using independent component analysis and continuous wavelet transformation. IEEE Trans. Biomed. Eng. 61 (8).

Hramov, Alexander E., et al., 2015. Wavelets in Neuroscience. Springer.

Kafiul Islam, Md, et al., 2016. Methods for artefact detection and removal from scalp EEG: a review. Neurophysiologie Clinique/Clinical Neurophysiol. 46, 287-305.

Kafiul Islam, Md, et al., 2016. A wavelet-based artefact reduction from scalp EEG for epileptic seizure detection. IEEE J. Biomed. Health Inform. 20 (5).

Keilson, M.J., et al., 1987. Ambulatory cassette EEG in absence epilepsy. Pediatr. Neurol 3, 273-276.

Kramer, G., et al., 1999. Sonification report: status of the field and research agenda. Tech. Rep., Int. Commun. Auditory Display.

Lofhede, J., et al., 2010. Automatic classification of back-ground EEG activity in healthy and sick neonates. J. Neural Eng. 7 (1), 016007.

Loui, P., et al., 2014. Rapidly learned identification of epileptic seizures from sonified EEG. Front. Human Neurosci. 8, 820.

MATLAB and Statistics and Machine Learning Toolbox Release 2017b. The MathWorks, Inc., Natick, Massachusetts, United States.

Molla, M.K.I., et al., 2012. Artefact suppression from EEG signals using data adaptive time domain filtering. Neurocomputing 97, 297-308.

Mormann, F., et al., 2000. Mean phase coherence as a measure for phase synchronization and its application to the EEG of epilepsy patients Elsevier. Physica D 144 (2000), 358-369.

Patel, A.X., et al., 2014. A wavelet method for modelling and despiking motion artefacts from resting-state fMRI time series. NeuroImage 95, 287-304.

Sachs, L., 1984. Applied Statistics: A Handbook of Techniques. Springer-Verlag, New York, pp. 253.

Swisher, C.B., 2017. QEEG in Seizure Detection. Continuous EEG monitoring Principles and Practice. Springer International Publishing Switzerland, pp. 191-214.

Temko, Andriy, et al., 2015. Clinical implementation of a neonatal seizure detection algorithm. Decision Support Systems 70, 86-96.

Temko, A., et al., 2017. Towards a personalized real-time diagnosis in neonatal seizure detection. IEEE J. Trans. Eng. Health Med. 5, 2800414.

Upadhyay, R., et al., 2016. A comparative study of feature ranking techniques for epileptic seizure detection using wavelet transform. Comput. Electr. Eng. 53, 163-176.

Urigüen, J.A., et al., 2015. EEG artefact removal - state-of-the-art and guidelines. J. Neural Eng. 12, 031001.

A. Väljamäe et al. (2013) «A Review of Real-Time EEG Sonification research» ICAD 2013 pp. 85-93.

Vidyaratne, Lasitha S., Iftekharuddin, Khan M., 2017. Real-time epileptic seizure detection using EEG. IEEE Trans. Neural Syst. Rehabil. Eng. 25 (11), 2146-2156.

ye Gao, H., 1998. Wavelet shrinkage denoising using the non-negative garrote. J. Comput. Graph. Statist. 7, 469-488.

Yoo, J., et al., 2013. An 8-channel scalable EEG acquisition SoC with patient-specific seizure classification and recording processor. IEEE J. Solid State Circuits 48 (1), 214-228.

Zeng, K., et al., 2016. Automatic detection of absence seizures with compressive sensing EEG. Neurocomputing 171, 497-502.

Zhu, D., et al., 2008. An ICA-based Method for Automatic Eye Blink Artefact Correction in Multi-Channel EEG. Proc. 5th International Conference on Information Technology and Application in Biomedicine, Shenzen, China, pp. 338-341. 